I molti territori della Repubblica fascista. Amministrazione e società nella RSI

\title{
«Nella sfera della propria
} a cura di Roberto Parisini, Roberta Mira e Toni legittima competenza o dietro ordini da me Rovatti

ISSN: 2282-4979 DOI: $10.12977 /$ ereview264 impartiti». La Rsi a Lucca, tra illusioni di governo autonomo e spoliazione delle risorse del territorio

Il presente saggio intende offrire una ricostruzione delle dinamiche del governo della Repubblica sociale italiana (Rsi) nella città di Lucca, un'esperienza breve e problematica ma caratterizzata, soprattutto inizialmente, da tensioni e aspirazioni a un governo effettivo del territorio. La seconda parte del saggio vuole offrire un originale spunto di ricerca sulla storia amministrativa della Rsi attraverso le vicende processuali legate ai crimini di collaborazionismo.

This essay intends to offer a description of the Italian Social Republic (Rsi) government practise in Lucca (Tuscany), a brief and problematic experience, that nevertheless was, above all in the first phase, marked by aspirations to the effective government of the territory. The second part of the essay aims to offer some ideas for an original line of research on the administrative history of Rsi, through the case-files related to crimes of collaborationism.

\section{Cenno introduttivo}

Questo saggio intende offrire una ricostruzione e un'interpretazione delle tensioni amministrative e delle pratiche di governo della Repubblica sociale italiana nella città di Lucca, tra 1'8 settembre 1943 e il 5 settembre 1944, ovvero fino al momento della liberazione della città. Si accennerà quindi solo rapidamente alle dinamiche della gestione dell'emergenza, alle vicende belliche e militari della Guardia nazionale repubblicana (Gnr) e alla violenza esercitata contro i civili e contro la Resistenza partigiana: sono questi ultimi, del resto, gli aspetti sui quali 
la storiografia attualmente disponibile si è maggiormente concentrata, anche per il caso di Lucca [Pardini 2001; Pesi 2013; Rossi 2006]. Il presente lavoro, infatti, si concentra piuttosto sulla ricostruzione degli aspetti amministrativi e di gestione del territorio. Certo è necessario specificare che quello della Rsi lucchese rappresenta un caso amministrativo molto particolare e decisamente critico. Anzitutto perché la formazione e l'evoluzione della vicenda in oggetto è strettamente connessa alle dimensioni medio-piccole della città; inoltre, perché l'esperienza del governo Rsi nella città di Lucca durò meno di un anno, dal settembre 1943 al 4 settembre 1944; infine, perché dovette fare i conti con una linea del fronte sempre più vicina, elemento che spinse i tedeschi a dichiarare il territorio zona di operazioni (assumendone quindi il pieno e diretto controllo) già nel luglio del 1944. Una storia amministrativa, dunque, molto particolare, sicuramente problematica e fragile, soprattutto se confrontata con altre esperienze di governo fascista repubblicano dell'Italia settentrionale.

Questo saggio è il risultato dell'utilizzo, in particolare, di due fonti d'archivio: le carte della Prefettura di Lucca relative al 1943-1945, e la documentazione processuale della Corte d'assise straordinaria (Cas) di Lucca', che si occupò della messa a processo dei crimini di collaborazionismo nazifascista. Oltre alle diverse altre fonti utilizzate, si ritiene che l'incrocio di queste due documentazioni possa contribuire ad illuminare i tratti di un effettivo tentativo di governo da parte della Rsi lucchese, pur nella sua effimera e problematica vicenda amministrativa.

\section{II contesto generale: gestire l'emergenza, collaborare alle operazioni belliche dell'alleato tedesco}

A Lucca, piccolo capoluogo di provincia nel nord della Toscana, la ripresa del potere da parte fascista dopo l'8 settembre 1943 avvenne in un contesto in cui già da diversi giorni i tedeschi avevano saldamente in mano il controllo della città, ottenuto senza incontrare nessun serio tentativo di resistenza. A quel punto, nel corso del settembre ' 43 , alcuni storici esponenti del fascismo locale intraprendono un tentativo piuttosto deciso di ricostituzione del Partito e di acquisizione e 
assorbimento della struttura amministrativa cittadina e provinciale. L'ingegnere Mario Piazzesi, federale a Lucca tra il 1938 e il 1942, in qualità di nuovo capo della provincia scalza il prefetto Marotta, lo allontana e ne assume le funzioni (nonostante quest'ultimo fosse ben visto dai tedeschi e avesse con loro buoni rapporti).

Il grande, cruciale, anzi potremmo dire dominante impegno dell'amministrazione di Piazzesi fu quello della gestione dell'emergenza, ovvero della situazione sociale ed economica legata al conflitto. Le carte della Prefettura di Lucca tra il 1943 e il 1945 tracimano di documenti relativi all'assistenza degli sfollati, alla gestione degli spazi abitativi per chi era rimasto senza casa, al razionamento dei generi alimentari. La provincia di Lucca accoglieva inoltre, già nel settembre 1943, alcune migliaia di sfollati da altre zone della Toscana e d'Italia [Lenzi 1995; Pesi 2010]². Nei mesi a cavallo tra 1943 e 1944, il capo della provincia fu effettivamente molto attivo nel cercare di far fronte a questa situazione. Tuttavia, non ottenne nessun tipo di supporto dalle autorità tedesche, interessate, più di ogni altra cosa, allo sfruttamento delle risorse della zona e allo smantellamento delle sue strutture e delle risorse industriali; su tutte, la Cucirini Cantoni Coats - importante azienda cittadina del settore tessile - e gli impianti della Società metallurgica italiana di Fornaci di Barga, località montana a nord del capoluogo. Lo smantellamento del patrimonio industriale fu però meno rilevante e meno impattante, a livello sociale, di quanto invece non sia stato, per una piccola comunità come quella di Lucca, lo sfruttamento di manodopera civile; in particolare, per la costruzione, nel corso del 1944, delle infrastrutture difensive della futura linea Gotica, e poi anche, in seguito, per l'invio di manodopera in Germania. La Todt, l'organizzazione che, in sinergia con la Wehrmacht, si occupava nei vari paesi occupati della costruzione di strade e infrastrutture connesse al proseguimento delle operazioni belliche, reclutò centinaia di civili lucchesi per la costruzione dei contrafforti difensivi della porzione di linea Gotica che, tra 1944 e 1945, avrebbe diviso la provincia di Lucca in una porzione meridionale già liberata dagli Alleati (compreso l'abitato del capoluogo) e in una settentrionale ancora in mano nazifascista. 


\section{3. "L'unica possibilità di rinascita per la nostra Patria». II tentativo di controllo e di gestione del territorio}

L'inedita carica di capo della provincia con la quale, sotto la Repubblica sociale, vennero riunite in un'unica figura di governo la carica di prefetto e la carica partitica del segretario federale del fascio, voleva costituire una sintesi di quella tensione totalitaria, di quella aspirazione alla completa simbiosi tra apparato istituzionale e pratica politica che il regime non era riuscito a realizzare fino al 1943. E voleva superare, nelle feroci e stremate ambizioni del Mussolini di Salò, quel dualismo - molto spesso rivelatosi conflittuale - tra prefetti e federali delle varie province. Il capo della provincia avrebbe dovuto essere non più solo l'occhio, ma adesso anche un vero e proprio braccio operativo del governo fascista su ogni lembo del territorio, e avrebbe dovuto gestire e dirigere ogni tratto dell'amministrazione civile e di polizia.

In virtù di questa nuova impostazione amministrativa, ai capi delle province veniva conferito il potere di nominare direttamente i podestà dei comuni con meno di 20.000 abitanti $^{3}$. Nella propaganda e nei proclami delle autorità della Rsi, ciò veniva descritto come una potente novità che avrebbe fatto discendere direttamente dal duce, per il tramite della nomina dei podestà da parte dei capi delle province, la conformazione delle amministrazioni locali e, dunque, avrebbe garantito l'effettivo controllo del territorio da parte del nuovo regime. Durante il Ventennio la nomina dei podestà spettava direttamente al re, su indicazione dei prefetti e del Ministero dell'Interno e sotto l'indirizzo e il controllo del Partito: dunque, già prima del 25 luglio 1943 i podestà erano generalmente sottoposti al controllo governativo in modo stringente. Si potrebbe quindi suggerire che, in realtà, questa "novità" della nomina dei podestà da parte dei capi delle province tendeva a delegare alla periferia l'individuazione, sul territorio, di qualcuno che potesse ricoprire la carica. Sembra perciò legittimo supporre che dietro questa misura sia possibile leggere un segno della debolezza del governo fascista repubblicano e, al contempo, della pulsione difensiva dei singoli spazi territoriali rispetto all'esterno in un momento di crisi lacerante della coesione identitaria, sociale e amministrativa delle comunità locali.

Nella realtà, inoltre, questa pretesa e questa potenzialità di controllo del territorio si scontravano con la situazione drammaticamente convulsa successiva all' 8 settembre e con le tensioni del contesto di guerra. Il capo della provincia di Lucca,

Archivio di Stato di Lucca (d'ora in poi ASLU), Prefettura 1943-1945, b. 4462, f. Ministero dell'Interno, II Ministero ai capi delle province, 17 marzo 1944. 
Mario Piazzesi, inizialmente si adoperò perché tutti gli amministratori che erano in carica al 25 luglio 1943, e che erano stati destituiti o allontanati durante i quarantacinque giorni badogliani, riprendessero il loro posto. Non fu cosa semplice: gran parte dei vecchi amministratori avevano lasciato la provincia, molti non avevano più intenzione di riprendere le loro funzioni. Tuttavia, tratto distintivo dell'operato di Piazzesi fu il tentativo di ricostruire un tessuto amministrativo vivo e, il più delle volte, non coincidente con il potere partitico: un elemento che pare di grande rilievo è infatti costituito dal fatto che, in seguito alle nomine fatte da Piazzesi, venne definita una lista di amministratori nella quale quasi mai l'amministrazione comunale coincideva con la guida del fascio locale. Piazzesi attribuì grande importanza al ruolo dei podestà della provincia, rivolgendosi molto più spesso a questi piuttosto che agli uomini del partito; e, con cadenza mensile, inviò a questi lunghi rapporti, i quali riportavano anche dettagliati elenchi delle prerogative e dei compiti che egli riteneva spettanti alle amministrazioni locali. Nel rapporto inviato da Piazzesi ai podestà il 25 febbraio 1944, il capo della provincia affermava che i titolari e gli impiegati degli uffici comunali «non devono fare politica, ma devono essere dei perfetti burocrati» [Pardini 2001, 231]. Inoltre, per quanto riguarda il caso specifico della città di Lucca, dopo un commissariamento durato cinque anni, il comune, per iniziativa di Piazzesi, venne nuovamente affidato a un podestà, il prof. Domenico Giannini, che andò a sostituire il commissario prefettizio Alpinolo Franci.

A proposito di questa attenzione di Piazzesi per il ruolo e le funzioni dei podestà, risulta davvero interessante una comunicazione inviata dal capo della provincia al comando tedesco il 10 maggio 1944. In quest'occasione, denunciando forti ingerenze nelle amministrazioni locali, Piazzesi scrive che i tedeschi spesso

[...] intervengono presso le Autorità comunali e si ingeriscono, a favore dei privati stessi, in questioni di carattere puramente amministrativo. Talvolta dispongono anche la modificazione o la sospensione di provvedimenti amministrativi, che l'Autorità Comunale adotta nella sfera della propria legittima competenza o dietro ordini da me impartiti [corsivo mio]. Ciò è doppiamente dannoso perché, mentre da una parte consente ad elementi spesso non raccomandabili di raggiungere le loro mire egoistiche, sfruttando l'Autorità germanica, alla quale alterano la verità, dall'altra parte discredita agli occhi dei cittadini gli organi dell'amministrazione comunale [corsivo mio] indebolendo l'efficacia della loro azionet.

Casi del genere, scrive Piazzesi, si sarebbero verificati in molte località. 
Ma è proprio a quest'altezza, all'inizio del maggio 1944, che si decide per lo spostamento di Piazzesi alla guida di un'altra Provincia, nel nord. Il 7 maggio, questi riceve un telegramma dal Ministero dell'Interno che lo informa del suo trasferimento alla guida di Piacenza, e della sua sostituzione, a Lucca, con Luigi Olivieri. Quest'ultimo, classe 1905, aquilano di nascita, medico, risiedeva a Lucca da alcuni anni; Seniore della Milizia, aveva combattuto sul fronte orientale ed era stato decorato per azioni di guerra; era stato podestà e commissario prefettizio di varie località aquilane negli anni Trenta. E' probabile che questa sostituzione sia da imputare, almeno in parte, alla volontà di spostare al nord le risorse umane considerate migliori, tra le quali anche gli uomini di governo dimostratisi più energici come, evidentemente, Piazzesi [Pardini 2001, 291-292]. Tuttavia, potrebbero aver pesato anche i problematici rapporti personali di Piazzesi con Alessandro Pavolini, potente gerarca nazionale ma, prima di tutto, "ras" del fascismo toscano [Pardini 2001, 60]. E' necessario ricordare, infatti, che Mario Piazzesi era considerato un uomo di potere a Lucca, e che era stato federale della città fino al 1942, anno in cui era stato poi allontanato dal vertice del potere politico cittadino in modo molto brusco e messo da parte con dinamiche a tutt'oggi non chiare. Nel suo Diario di uno squadrista toscano, Piazzesi ha sostenuto che la sua caduta politica nel 1942 sarebbe da imputare ai contrasti interni al fascismo toscano; in particolare, sembra verosimile che Pavolini fosse preoccupato per alcune voci riguardanti una possibile nomina di Piazzesi a Federale di Firenze, che il "ras" toscano considerava un suo feudo personale [Piazzesi 1981, 11-44]. Il fatto che, nel settembre 1943, Piazzesi fosse stato chiamato a riprendere il controllo di Lucca può essere motivato con ragioni di automatismo e immediatezza in una fase delicata come quella successiva all' 8 settembre: si scelse l'uomo che, in tempi recenti, aveva tenuto più a lungo e con migliori risultati il controllo politico sulla città.

La gestione del nuovo capo della provincia Olivieri, insediatosi ufficialmente il 14 maggio 1944, pare essere caratterizzata da una ben più debole attività amministrativa e di governo, e da un ripiegamento via via più esplicito rispetto alle necessità dettate dall'avvicinarsi del fronte e dalle esigenze militari tedesche. In alcuni casi, tuttavia, egli fece giungere al comando germanico alcune rimostranze in merito ad aggressioni e a violenti atteggiamenti dei soldati della Wehrmacht verso i civili italiani5. In data 29 maggio 1944, ad esempio, Olivieri scrive al Comandante di zona tedesco per denunciare un fatto avvenuto su un convoglio ferroviario: dei soldati tedeschi avrebbero minacciato il conduttore a seguito di 
un sobbalzo del mezzo, imputandogliene la causa; sarebbe intervenuto in sua difesa un brigadiere della Guardia nazionale repubblicana (Gnr); i soldati tedeschi avrebbero fatto scendere quest'ultimo dal convoglio, in campagna, e lo avrebbero picchiato. Scrive a tale proposito Olivieri:

L'episodio ha provocato viva indignazione fra tutti i viaggiatori, i quali si sono astenuti dall'intervenire per un doveroso riguardo alle Forze armate tedesche [corsivo mio]. Ho voluto sottoporvi il fatto alla vostra attenzione perché il ripetersi di tali incidenti del genere potrebbe turbare la cordialità dei rapporti, che finora è esistita fra le forze armate tedesche e la popolazione, ed indurre la popolazione stessa a reagire contro i provocatori ${ }^{6}$.

Tuttavia, l'autorità di Olivieri ha vita molto breve. La città di Lucca e la piana circostante vengono liberate tra il 4 e il 5 settembre 1944, ma l'esperienza locale della Repubblica sociale era già finita, di fatto, con l'inizio del luglio dello stesso anno, quando il territorio era stato dichiarato "zona di operazioni" e, dunque, posto sotto il completo controllo dell'autorità tedesca. Siamo perciò di fronte ad un'esperienza politico-amministrativa che ebbe vita brevissima (metà settembre '43-inizio luglio '44), ed ebbe pochi mesi a disposizione per darsi uomini e strutture e per assumere un potere che, nello stesso momento in cui cercava di darsi una sua autonoma solidità e una propria sostanza, con il rapido avvicinarsi del fronte veniva progressivamente assorbito e poi esautorato dalle esigenze militari delle truppe germaniche.

Deve poi essere sottolineato come, in lucchesia, specie a partire dalla tarda primavera del 1944, i tedeschi vedessero nelle autorità militari fasciste il referente principale, in particolare nella persona di Bruno Messori, capo della Guardia nazionale repubblicana lucchese. Nel giugno 1944, poi, in contemporanea con la creazione delle Brigate nere, la visita di Pavolini a Lucca è decisiva per la definitiva militarizzazione del potere fascista su quel territorio: nei fatti, egli sollevò dall'incarico Luigi Olivieri, per dare il comando a Idreno Utimpergher, uomo legato personalmente e fortemente a Pavolini. Nel giugno 1944, Utimpergher accompagnò Pavolini in viaggio nell' Italia centrale, nei territori dove la Rsi sembrava essere maggiormente in difficoltà. Su proposta di Pavolini, Utimpergher si fermò a Lucca e guidò la XXXVI Brigata nera "Mussolini", in seguito denominata "Natale Piagentini" (in omaggio a un milite caduto); alla brigata venne poi attribuita la partecipazione a numerosi episodi di violenza contro i civili. A partire da questo momento, non si può più parlare, per Lucca, di una struttura ammi- 
nistrativa funzionante; sembra più lecito parlare, invece, di un potere autoritario militare in stretto contatto - anzi, in contiguità - con le forze armate tedesche. Sembra dunque legittimo affermare che, prima del giugno 1944 vi sia stato, in particolare da parte del primo capo della provincia Piazzesi, un tentativo di controllo e di governo effettivo del territorio; e che lo stesso Piazzesi abbia rivendicato l'importanza e la necessità di autonomia delle «autorità comunali». Tuttavia, a Lucca pesarono sia l'approssimarsi della linea del fronte e, dunque, l'intensificarsi dello schiacciamento da parte dell'autorità militare tedesca, sia, molto probabilmente, anche l'influenza degli alti gerarchi toscani, come Pavolini, e l'imposizione di una concezione militaristica del potere nella repubblica fascista. L'idea che la forza militare fosse un elemento cruciale per l'energia vitale dello Stato di Salò non era estranea neppure a Piazzesi; tuttavia, il suo approccio appare sensibilmente diverso rispetto a quello di Pavolini. Ad esempio, alla fine di dicembre del 1943, protestando con il comando tedesco per il fatto che dall'Organizzazione Todt venissero reclutati troppi giovani che invece avrebbero dovuto svolgere il servizio militare «nell'esercito italiano», Piazzesi fece risaltare l'elemento dell'appartenenza nazionale sopra ogni altra cosa: servire il proprio esercito nazionale non poteva essere visto dalle autorità tedesche come secondario rispetto alle esigenze di reclutamento di manodopera. In quell'occasione, Piazzesi ebbe anche a dire che «la ricostruzione dell'esercito italiano rappresenta l'unica possibilità di rinascita della nostra Patria»? Se Piazzesi parlava di «esercito italiano», Pavolini aveva in mente piuttosto un «esercito fascista», che tentò di concretizzare con la creazione delle Brigate nere. Sembra dunque possibile individuare un'importante differenza di approccio tra i due e rintracciare un tratto distintivo del fascismo repubblicano di Piazzesi.

\section{Tra amministrazione autonoma e spoliazione del territorio: meccanismi della Rsi lucchese nelle carte processuali per reati di collaborazionismo}

Saranno adesso portate all'attenzione dell'indagine due vicende processuali sottoposte al lavoro della Corte d'assise straordinaria (Cas) di Lucca, dunque appartenenti a quella "giustizia di transizione" contro i reati fascisti e di collaborazionismo, giudicati nell'immediato secondo dopoguerra. Vengono qui analizzate 
perché si ritiene possano restituire il clima del fascismo repubblicano e della Rsi a Lucca e perché si vogliono suggerire nuove possibilità di indagine storiografica sulla Rsi attraverso lo studio delle vicende processuali della giustizia di transizione. I procedimenti penali per collaborazionismo nazifascista sono stati fino ad ora utilizzati dalla storiografia principalmente per trattare gli avvenimenti e le dinamiche della violenza esercitata del fascismo repubblicano sulla popolazione civile nel 1943-1945. Si ritiene invece che queste carte, in particolare quelle relative a personaggi non particolarmente noti, e tuttavia ben inseriti nel tessuto del potere della Rsi, possano aiutare a ricostruire le vicende della Repubblica sociale anche come storia di un tentativo di amministrazione e di controllo del territorio. Non essendo in questa sede interessati alla giustizia di transizione in quanto tale, non saranno trattati gli aspetti maggiormente tecnici legati alla dinamica processuale ma, piuttosto, gli elementi riguardanti il ruolo politico e amministrativo dei soggetti in questione, emersi in sede di indagine e di dibattimento.

La prima di queste due vicende processuali riguarda Mario Rossi ${ }^{8}$. Questi, nato a Pavia nel 1894, era da poco tempo domiciliato a Lucca, ma risultava essere ispettore federale del fascio ed essersi iscritto fin da subito al fascio repubblicano. Rossi verrà imputato, nel giugno 1945, del reato di collaborazionismo «per avere in Lucca, dopo 1'8 settembre '43 [...] favorito i disegni del tedesco invasore accettando ed esercitando la carica di ispettore federale del Pfr [Partito fascista repubblicano]», e per aver facilitato la consegna dell'argento giacente presso la Banca d'Italia e la sottrazione, dalla stessa, di fondi del conto corrente della Prefettura. Dagli atti del processo, Rossi risulterà anche essere stato membro di una commissione (istituita presso il locale Ufficio del lavoro/Ufficio di collocamento) per l'invio degli operai in Germania, di cui parleremo più avanti in merito al secondo procedimento qui preso in esame.

La vicenda processuale di Mario Rossi parte il 2 marzo 1945 quando Pietro Mori, delegato provinciale della Commissione di epurazione di Lucca [Bennati 2016a e 2016b], invia alla Procura del Regno un foglio con allegate varie denunce e testimonianze a carico di Mario Rossi: «Per il procedimento penale contro la persona in oggetto, per il reato di cui all'articolo 5 DLL 27.7.44 n 159, si trasmettono gli atti. Prego far conoscere a suo tempo l'esito del procedimento». Le denunce e le dichiarazioni in oggetto sono raccolte tra il dicembre ' 44 e il marzo ' $45 . \mathrm{La}$ denuncia alla base di tutto provenne dalla stessa Commissione interna per l'epurazione istituita presso la Banca d'Italia all'indomani della Liberazione: i membri 
della commissione, firmatari del documento (tra gli altri, figurano il cassiere della tesoreria della banca e il segretario di tesoreria) si richiamano all'esposto "presentato il 9 settembre 44 [pochi giorni dopo la liberazione di Lucca] che provocò l'allontanamento dall'Ufficio del Sig. Rossi»; gli stessi aggiungono che, nella compilazione della sua scheda personale di dipendente della filiale, il Rossi ha omesso «volutamente la qualifica di ispettore federale del PFR e di membro della commissione per l'invio di operai in Germania». Ma i membri della commissione interna di epurazione vanno oltre:

si invita inoltre la Commissione [per l'Epurazione] a voler chiedere al direttore della Banca d'Italia in base a quali ordini egli consegnò l'argento giacente presso la filiale della Banca d'Italia alle Autorità fasciste [...], ciò perché si ha fondato sospetto che tale consegna sia avvenuta in seguito a denunzia del predetto Rossi e tale sospetto [...] si estende anche ai fondi del conto corrente speciale della Prefettura prelevati dietro segnalazione giornaliera fatta dal signor Rossi agli allora Capi della provincia Olivieri e Utimpergher.

Per quanto riguarda la qualifica del Rossi come «membro della commissione per l'invio di forza lavoro in Germania», i membri della commissione epurativa della banca attestano «che tale qualifica era puramente formale ma che veniva esplicata con alacrità», visto che nei locali stessi della banca di Italia si presentavano quotidianamente informatori e i familiari di coloro che venivano spediti a lavorare in Germania, i quali spesso supplicavano perché i propri figli non venissero sottoposti alla misura: «di fronte alle richieste, il Rossi si dimostrava più volte irremovibile». Il 2 marzo 1945, dalla Banca d'Italia giunge alla Commissione d'inchiesta presso il Comitato di liberazione nazionale (Cln) di Lucca un altro foglio di denuncia contro il Rossi firmato da ventiquattro persone, comprendenti i membri della commissione interna di epurazione della banca. Di questi, sedici sono impiegati.

Altro documento interessante dell'incartamento processuale è la denuncia di Alpinolo Franci, vice prefetto di Lucca, depositata alla delegazione di Pietro Mori il 22 febbraio 1945. Franci ricorda che qualche tempo dopo l'avvicendamento tra Piazzesi e Olivieri a capo della provincia, nel maggio 1944, apprese da quest'ultimo che il ministro dell'agricoltura della Rsi, Edoardo Moroni - cresciuto a Lucca e con rapporti importanti con la città - aveva dato ordine a Olivieri affinché tutti i beni dello Stato esistenti nella provincia venissero portati al nord. Olivieri sarebbe riuscito, proprio attraverso il Rossi, a venire a conoscenza dell'esistenza, presso la Banca d'Italia di Lucca, di un conto della Prefettura di oltre 40 milioni: al Rossi, ispettore presso la tesoreria della banca, era stato dunque affidato il compito di occuparsi del prelevamento. 
Il Franci, temendo per i fondi presenti sul conto della Prefettura presso la Banca d'Italia di Lucca, avrebbe dunque parlato con il ragioniere capo Ermete Grossi e con il ragioniere Sansoni, entrambi della Prefettura, «per studiare il modo di evitare questo non lieve danno alle pubbliche finanze». Il Grossi si sarebbe dunque recato dal direttore della filiale lucchese della banca, Albi Marini. Una volta appurata «l'assoluta impossibilità di giovarci della Banca d'Italia per i nostri fini», i tre decisero di

provvedere al pagamento (con opportuni ordinativi a favore della Direzione Postale) di quanto esisteva in cassa ed era inerente al servizio dei sussidi militari; quindi fatti gli ordinativi per tutti i 40 milioni, per una parte minore si sarebbe corrisposta la somma e per il resto si sarebbe tenuta bloccata al fine di conservare l'ammontare per le necessità successive.

Con questo complesso meccanismo i tre speravano di riuscire di impedire lo spostamento al nord di gran parte delle risorse monetarie presenti nella filiale.

Tuttavia, per un'operazione del genere, il viceprefetto Franci doveva ottenere l'assenso del capo della provincia Olivieri. Durante il colloquio tra i due, l'Olivieri chiese se, fatti questi pagamenti alla direzione postale, fossero rimasti altri fondi, al che Franci avrebbe risposto affermativamente, ma in modo volutamente approssimativo. Olivieri «rimase perplesso, e chiamò al telefono il Rag. Rossi, controllore della Tesoreria Provinciale», il quale gli confermò che presso il conto della Prefettura si trovavano 42 milioni di lire: 1'Olivieri dunque si rifiutò di aderire alla richiesta del viceprefetto circa l'approvazione degli ordinativi e, soltanto dopo le insistenze di quest'ultimo, il capo della provincia accettò di corrispondere alla Direzione Postale la cifra di 12 milioni, non di più. Tuttavia, nei giorni successivi, un uomo della Prefettura, d'accordo con Franci, con l'aiuto di due dipendenti della filiale, sarebbe riuscito a far trasferire, attraverso un complesso meccanismo di pagamenti, buona parte dei 42 milioni, mettendoli in salvo; tanto che i fondi si sarebbero ridotti a 8 milioni e l'Olivieri, partito poi improvvisamente, non sarebbe riuscito ad asportare nulla del denaro della Prefettura.

Durante l'istruttoria è interrogato, tra gli altri, il direttore della filiale della banca, Albi Marini. In merito alla questione del conto corrente della Prefettura, Marini afferma che in quel tempo il Rossi, informando la Prefettura, avrebbe compiuto «né più né meno che il suo dovere». Il prelevamento di cui doveva occuparsi il Rossi sarebbe stato regolare; afferma infatti il direttore:

poiché le autorità costituite in quel tempo, in questa parte del territorio, erano quelle della sedicente repubblica sociale, il prelevamento, avvenuto con ordinanza del Capo della Provincia, che aveva pieni poteri, lo ritengo se non ortodosso, regolare $[\ldots]$. 
Ovviamente, questa fu anche la posizione assunta dal Rossi per difendere il suo operato: durante il suo interrogatorio sostenne più volte di aver agito correttamente, giacché, disse, «la tesoreria è tenuta a comunicare alla Prefettura, con un modulo espressamente istituito, i pagamenti, gli introiti e le rimanenze di cassa di un conto corrente intestato alla Prefettura».

Se il prelevamento dei fondi della Prefettura non sembra essere andato a buon fine, riuscito pienamente sembra invece essere stato quello dell'argento dal $\mathrm{ca}$ veau della banca. Questo è quanto risulta da un verbale dell'operazione di prelevamento, portato come prova documentaria al processo (una delle rarissime prove documentarie in questa tipologia di procedimenti). L'operazione è avvenuta all'alba del 21 giugno 1944, alla presenza delle locali autorità civili e di polizia. Si informa che «In esecuzione dell'ordinanza 20 giugno 1944 n 906 Gab. del Capo della Provincia di Lucca», al vicecommissario Cavilli è stato consegnato «tutto l'argento esistente nelle Casse della Succursale», e che «per disposizione dei Ministeri delle Finanze e del Tesoro, doveva costituire riserva e, quindi, non spendibile». L'ammontare dell'argento in lire risulta essere pari a 7.894.000 lire, costituente la voce «argento fuori dotazione», mentre pari a 10.000 lire risulta essere quello «in dotazione». Nel verbale dell'operazione, si informa anche che l'argento è contenuto «in $\mathrm{n}^{\circ} 1.580$ sacchetti di tela, contenenti ciascuno monete per l'ammontare di lire cinquemila».

La vicenda dell'argento della filiale lucchese della Banca d'Italia fece partire, subito dopo la Liberazione, una serie di indagini estese al resto della regione, per accertare se vi fossero stati altri fatti del genere. Le Questure di Firenze, di Siena, di Pisa, di Pistoia e di Arezzo risposero che in nessuna di quelle località era accaduto niente del genere riguardo alle riserve di argento delle filiali di Banca d'Italia.

Nel corso del procedimento contro Mario Rossi si è parlato di un suo coinvolgimento nelle pratiche dell'Ufficio del lavoro. La Corte d'assise straordinaria di Lucca si occupò anche, nelle stesse settimane del processo contro Rossi, del procedimento contro Ivano Ricci e altre quattro persone ${ }^{9}$, membri di una commissione deputata alla precettazione di manodopera per i tedeschi e all'invio di manodopera in Germania. Ricci, cinquantenne livornese, classe 1896, ex militare, commerciante, era poi sfollato a Lucca a causa dei bombardamenti. All'indomani della Liberazione, verrà imputato di collaborazionismo per il suo ruolo attivo 
di «collocatore di manodopera per il nemico e alla deportazione di questa, e per privazione della libertà delle persone precettate e deportate».

Le prime fasi delle indagini, che mirano a ricostruire i meccanismi e a punire gli interpreti di questa rete adibita alla precettazione di manodopera, risalgono al febbraio-marzo 1945. Il nuovo questore della Lucca liberata, Cocci, stende un rapporto su Ivano Ricci il 6 marzo 1945. Cocci, in seguito alle indagini svolte su indicazione del delegato per l'epurazione Mori e del Cln, scrive: «risulta che Ricci sia in questa città dal 28 dicembre 1940, proveniente da Livorno, in qualità di capo dell'Ufficio provinciale del lavoro, inviso alla popolazione». Descritto dal questore come un fascista di vecchia data, squadrista, dopo l'8 settembre questi «rimaneva al suo posto di lavoro continuando a manifestare sentimenti filofascisti e filo nazisti ed aderendo, con entusiasmo, al fascio repubblicano». Durante questo periodo, il Ricci,

a capo dell'Ufficio provinciale del lavoro per il reclutamento della manodopera da inviare in Germania [...] sguinzagliava nelle campagne i militi della costituita compagnia del lavoro alle sue dipendenze allo scopo di effettuare rastrellamenti di persone e procedendo al fermo di coloro che, precedentemente invitati con la cartolina precetto, non si erano presentati per l'invio obbligatorio in Germania. $[\ldots]$ le sue gesta e i suoi mezzi coercitivi $[\ldots]$ nei confronti della classe operaia, perseguitata dai nazifascisti, sono note alla cittadinanza, che indignata desidera che nei confronti del medesimo siano adottati provvedimenti di rigore.

Riguardo alla reperibilità del Ricci durante le indagini, Cocci ammette che da qualche tempo non lo si vede più in città e che, secondo alcune voci, si potrebbe trovare in condizioni di malferma salute nei pressi di Capannori - cittadina poco ad est di Lucca, dove il Ricci aveva la residenza. Un paio di mesi dopo il rapporto del questore, il 7 maggio i carabinieri di San Concordio, in merito all'ordine di cattura emanato nei confronti del Ricci, informano che hanno avuto notizia che il soggetto, «in modo non dubbio [...], pochi giorni prima della liberazione della città di Lucca ripiegava a Nord con la propria famiglia, e non ha fatto ritorno a [Capannori]».

Sul conto degli imputati vengono raccolte denunce e testimonianze importanti, alcune delle quali provenienti da personaggi illustri della guerra di liberazione. Ad esempio Mario Bonacchi, il quale, oltre alla sua testimonianza (autorevole, poiché questi era stato uno degli esponenti di spicco della Resistenza in provincia di Lucca), reca con sé anche un documento probatorio che risulterà preziosissimo per l'accusa; il Bonacchi ne era entrato in possesso perché, all'indomani della Liberazione, aveva ricoperto la carica di direttore dell'Ufficio provinciale del lavoro, proprio la stessa che era stata del Ricci. Il documento ritrovato da Bonacchi è una nota, ritornatagli in ufficio «poiché respinta dalle Poste», con l'intestazione 
«Commissariato nazionale del lavoro - Ufficio provinciale di collocamento di Lucca - Direzione collocamento», con oggetto «Precettazione», datata 22 maggio 1944, a firma del «Cap. Ricci Ivano, Direttore Provinciale»: nel documento, Ricci informa il Commissariato del lavoro, con sede a Verona, dei risultati e dei numeri della prima fase del lavoro di precettazione, chiedendo una sospensione dell'attività. Ricci riconosce che si sono avuti molti ostacoli a livello organizzativo nel lavoro di precettazione e di ricerca dei non presentati, anzitutto per la mancanza di mezzi, e che tiene a rendere noto che per il momento «si è precettato solo i cittadini di questo comune [Lucca] e di Capannori». Scrive poi il Ricci che la sospensione non dovrà però durare molto, «ma solo il tempo necessario perché la polizia del Lavoro possa rastrellare un notevole numero di renitenti». Il documento è firmato da Ricci, e costituirà in aula una prova pesantissima a suo carico. Al 22 maggio 1944, sarebbero 3.333 i cittadini precettati, in generale, per il lavoro obbligatorio; di questi, solo 1.528 si sarebbero effettivamente presentati a rapporto.

Per quanto riguarda la precettazione specifica di lavoratori da inviare in Germania, alla documentazione processuale sono accluse anche diverse cartoline precetto portate alle autorità inquirenti da alcuni cittadini, con la lettera di invito a firma di Ricci. Meritano citazione alcune parti del modello di lettera-precetto: si informa il futuro «lavoratore» che «per ragioni tecniche» non può ancora essergli detto che tipo di lavoro farà e non può ancora essergli consegnato il contratto, ma che «il trattamento è però garantito perfettamente uguale a quello del lavoratore tedesco della stessa categoria». Ancora, si informava:

[...] poiché può essere che nell'azienda vi siano lavoratori di altre nazionalità e prigionieri di guerra, tu non dovrai assolutamente avere rapporti con essi, in modo da salvaguardare la tua dignità di libero lavoratore italiano [corsivo mio]. Ricorda sempre che sei un Italiano della Repubblica Sociale Fascista, che compie il proprio dovere verso la Patria, come il soldato che combatte al fronte.

Al termine del modulo si informava il precettato delle gravi conseguenze che avrebbero colpito lui e la sua famiglia nel caso non si fosse presentato.

Come in altri casi, non mancano neppure testimonianze di persone che, contrariamente alle accuse che pesano sull'imputato, sostengono di essere state anzi da lui aiutate a evitare la precettazione. Il 22 ottobre 1945 viene redatto, quasi certamente su iniziativa dell'avvocato difensore del Ricci, un foglio manoscritto con due sottoscrizioni collettive e due dichiarazioni singole. Natalino G., «operaio della Todt», afferma che gli fu consigliato dal Ricci di lasciare immediatamente il lavoro per evitare di essere precettato; Guerrino G., afferma che il Ricci gli avrebbe consigliato di lasciare subito il lavoro, sempre per lo stesso motivo. 
Interessante il fatto che entrambi i soggetti di queste dichiarazioni abitino a Tempagnano (Capannori), località di residenza di Ricci prima della fuga al nord. Le due sottoscrizioni collettive, sono firmate da una quarantina di persone, sempre di Tempagnano; va notato che anche queste due, come le precedenti dichiarazioni singole, sono identiche tra loro.

Ma le autorità inquirenti stanno cercando di ricostruire un'attività istituzionale di precettazione che certo non comprendeva solo il Ricci. Il 24 dicembre 1945, il questore di Lucca scrive un rapporto (oggetto: «Commissariato Provinciale del lavoro») in cui fa il punto della situazione investigativa. Si scrive che «a Lucca, in Piazza Bernardini aveva sede il Commissariato Provinciale per la precettazione obbligatoria del lavoro», già ufficio di collocamento, che nel febbraio '44 fu unificato e dato in affidamento al Ricci. Presso l'Ufficio aveva sede una Commissione composta di diritto dai segretari delle Unioni sindacali, settori del commercio, dell'industria, dell'agricoltura e dal fiduciario del pubblico impiego, rappresentati rispettivamente: per il commercio, da Bruno Burchi (nato a Firenze nel 1896), venuto a Lucca da Viareggio nell'ottobre del 1941; per l'agricoltura, da Guerrino Bucci (Terni, 1894), venuto a Lucca da Pietrasanta; per l'industria, da Mario Baldi, «di anni 43 circa, non meglio identificato»; infine, come fiduciario del pubblico impiego, Mario Rossi, «già capo ufficio tesoreria della Banca d'Italia».

L'inquadramento della commissione nel tessuto amministrativo non risulterà del tutto chiaro nemmeno dal testo della sentenza, ma è evidente come essa fosse sorta dall'Ufficio di collocamento e dall'ufficio del lavoro.

Si ritiene importante sottolineare che, dall'incartamento processuale, risulta che a partire dal luglio 1944, ovvero dal momento in cui la zona di Lucca viene dichiarata zona di operazioni, il lavoro della commissione si arresta e ad esso si sostituisce il rastrellamento tedesco, del tutto indiscriminato.

Il documento inviato dal Ricci al Commissario nazionale del lavoro di Verona (documento il quale, come nel caso del Rossi, costituisce una rara prova documentale), verrà ritenuto dalla corte una prova schiacciante di colpevolezza e di attività criminale pienamente collaborazionistica. Nelle motivazioni della sentenza della Cas di Lucca, che 1'8 febbraio 1946 condannerà il Ricci a 25 anni di reclusione, si legge come questi venne posto a capo dell'Ufficio provinciale di collocamento, e che «detto ufficio fu creato per facilitare il reclutamento di manodopera da inviare in Germania»; che il reclutamento sarebbe avvenuto, in un primo tempo, in maniera coercitiva mediante precettazione e, in un secondo momento (dal luglio 1944), mediante rastrellamenti: 
[...] la precettazione all'inizio veniva fatta in base agli elenchi degli operai disoccupati, poi in base agli schedari degli operai e infine su elenchi anagrafici per classi. In caso di mancata presentazione, gli operai venivano arrestati $[\ldots]$.

A Firenze, i precettati e gli arrestati venivano poi consegnati all'autorità tedesca. La corte continua affermando che, a detta dei testimoni, il Ricci non fu affatto obbligato a ricoprire il ruolo di direttore dell'Ufficio durante il periodo della precettazione, e che anzi «fu lui stesso che sollecitò la nomina»; egli «fu sempre intransigente e inumano».

Continua la corte nella sentenza:

aver partecipato direttamente e direttivamente al sistema di precettazione rende colpevole non della forma più contestata di collaborazionismo, ovvero quella legata all'art $56^{10} \mathrm{del} \mathrm{cpmg}$ [codice penale militare di guerra], ma sibbene a quella politica cioè [a] quella prevista dalla legge speciale in relazione all'art. 58 [...].

Inoltre, affermano il giudice e i giurati, a sua discolpa il Ricci non può sostenere di essersi limitato ad eseguire ordini superiori del capo della provincia e, dunque, di non avere responsabilità penali, dal momento che

tutte le organizzazioni e le istituzioni del cosiddetto governo repubblicano devono considerarsi illegittime e quindi nei loro riguardi non si può parlare di gerarchia e di ordini legittimi e quindi di obbedienza gerarchica [corsivo mio]. E tutto ciò senza osservare che spesso il Ricci [...] agiva indipendentemente e contro gli ordini superiori.

Il caso di Ivano Ricci apre lo sguardo su un aspetto molto rilevante dell'occupazione e del collaborazionismo nazifascista, ovvero quello della precettazione di manodopera civile per il tedesco invasore, in particolare quella da inviare in Germania. Si tratta di un fenomeno che, nonostante abbia coinvolto un numero consistente di persone (si parla qui solo della manodopera civile, senza contare gli internati militari), è stato finora relativamente poco studiato [Mantelli 2006]. Non sembra ancora possibile stabilire, con esattezza, quanti cittadini di Lucca e provincia siano stati effettivamente sottoposti a questo trattamento e poi inviati in Germania. Sappiamo però, da un documento del marzo 1944, che l'ufficio di

Nelle imputazioni e nelle sentenze per reati di collaborazionismo, gli articoli del Codice penale militare di guerra (Cpmg) ai quali le Cas fecero più spesso riferimento furono l'art. 51 (aiuto al nemico nelle sue operazioni militari,fino al 1994 punibile con la morte secondo il (pmg), I'art. 54 (aiuto al nemico in generale, punibile con la morte o con pene minori se l'aiuto non aveva avuto effetti particolari) o l'art. 58 (aiuto al nemico al fine dei suoi disegni politici, punibile con la reclusione da dieci a venti anni). La corte della Cas di Lucca in alcuni casi fece però ricorso anche all'art. 56 («Comunicazione illecita con il nemico senza il fine di favorirlo», punibile con la reclusione da uno a sette anni oppure fino a dieci se il fatto si era ripetuto più volte), un articolo che permetteva di individuare una forma meno grave di intelligenza con il nemico. 
Ricci aveva compilato un elenco di 161 persone precettate ${ }^{11}$. Sembra possibile fare una considerazione sulle caratteristiche e sulle finalità di queste operazioni, almeno dal punto di vista delle autorità del fascismo repubblicano: fino al luglio 1944, ovvero fino a quando il reclutamento rimase in mano all'ufficio di Ricci, e fino a quando le autorità germaniche non procedettero ai rastrellamenti indiscriminati, vi fu una gestione delle chiamate che appare ragionata, studiata. L'elenco dei 161, infatti, era comprensivo dell'indicazione della qualifica professionale di ogni precettato: dalla lettura dell'elenco, si può ricavare come si trattasse principalmente di operai, manovali; o, ancora, di persone disoccupate. Anche il gran numero di testimonianze a favore del Ricci, testimonianze di persone che dichiarano di essere state scartate dalla precettazione, potrebbe supportare l'ipotesi di un reclutamento di manodopera per la Germania inteso anche come possibilità di compiere un piccolo "repulisti" sociale e morale della comunità cittadina, eliminando i soggetti ritenuti maggiormente disturbanti e potenzialmente pericolosi.

\section{Conclusioni}

Il fascismo repubblicano lucchese ha costituito un' esperienza molto breve e problematica, ma che può forse essere letta definendo una scansione in due segmenti temporali: una prima parte, quella caratterizzata dal governo di Mario Piazzesi (e da una maggior lontananza dal fronte e dalle operazioni belliche), nella quale si tentò di esercitare una forma di governo locale effettivamente autonomo; una seconda parte, che inizia con il maggio 1944 e con l'insediamento di Olivieri alla guida della provincia, contraddistinta da una maggior impotenza verso l'alleato tedesco e da un esautoramento di fatto delle funzioni dell'amministrazione italiana. E non deve essere casuale che la vicenda delle ricchezze delle Banca d'Italia trasportate al nord, ma anche quella del reclutamento coatto di manodopera per i tedeschi, siano iniziate e si siano svolte (almeno nella loro fase decisiva e culminante) nelle settimane del passaggio dalla prima alla seconda di queste due brevi stagioni. Entrambe le vicende giudiziarie analizzate descrivono un processo d'impoverimento e di privazione delle risorse (economiche e umane) del territorio e della comunità, da parte di un fascismo repubblicano che evidentemente cominciava a pensarsi già oltre la linea Gotica. Tuttavia, raccontano anche di una Repubblica sociale la quale, pur nella sua tensione finale, caratterizzata essenzialmente dalla razzia e della violenza, aspirava comunque ad agire definendo 
strutture e commissioni, inquadrando la sua attività all'interno di cariche e di titolature, alla ricerca disperata di legittimità istituzionale.

Inoltre, si ritiene che possa essere fatta una considerazione importante riguardo alla provenienza e ai profili biografici dei protagonisti di queste vicende. Mario Piazzesi, determinato nel suo tentativo di gestione effettiva del territorio, seppur nato a Forlì risiedeva a Lucca da molti anni, e aveva in Lucca radici e legami saldissimi. Luigi Olivieri (il secondo capo della provincia) era aquilano e domiciliato a Lucca solo da poco tempo, e pure i due protagonisti delle vicende processuali descritte, Mario Rossi e Ivano Ricci, giungevano il primo da Pavia e il secondo da Livorno, arrivati a Lucca solo durante la temperie della guerra. Stesso discorso può essere fatto anche per gli altri membri della commissione per l'invio dei lavoratori in Germania. Il diverso atteggiamento di chi tenne il potere sotto Salò, entro i molti terrori della Rsi, può essere motivato anche da questo aspetto: il legame più o meno stretto di questi uomini con una determinata località e comunità. Quella della Rsi, del resto, è anche la storia di politici e amministratori in movimento, mandati a esercitare un potere prima su di un territorio e poi su di un altro.

Per quanto riguarda, nello specifico, l'analisi delle due vicende processuali portate all'attenzione, si vuole qui suggerire e stimolare l'idea che si possa guardare alla storia della Rsi attraverso la lente della giustizia di transizione e dei processi per collaborazionismo. Ma non tanto e non più solo rispetto alle vicende processuali riguardanti le stragi e i gravissimi eventi di sangue della guerra civile del 1943-1945; ma, piuttosto, prendendo in esame procedimenti penali apparentemente minori e, tuttavia, capaci di gettare una luce sulle forme e sulle dinamiche dell'amministrazione e della gestione del territorio.

La Repubblica sociale italiana è stata tradizionalmente interpretata, dalla storiografia, come un mero "stato fantoccio", un semplice braccio operativo della violenza nazifascista. Tuttavia, sembra necessario riconoscere che lo studio è stato fino ad oggi condotto a partire da - e come excursus - della storia della lotta partigiana e del conflitto contro l'occupante tedesco e i suoi collaboratori fascisti: un punto di vista che non può che finire per cogliere massimamente - quando non esclusivamente - il carattere di forte subordinazione della Rsi rispetto agli agenti intorno e contro essa operanti. La Repubblica sociale venne pensata e architettata anzitutto dai tedeschi; ma questi ne permisero l'istituzione non solo con l'obiettivo di servirsene per un controllo poliziesco, paramilitare e repressivo del territorio, ma anche per poterne meglio utilizzare le risorse, umane e materiali, ai fini dello sforzo bellico, auspicandosi quindi di poter sfruttare un apparato amministrativo italiano più facilmente radicato sul territorio e inserito nelle comunità. 
Anche ammettendo che la genesi della Rsi tragga la sua origine e sia indissolubilmente connessa con i piani e le strategie tedesche, esiste dunque una dimensione amministrativa della Repubblica sociale, all'interno della quale le autorità e gli uomini del fascismo repubblicano operarono con modalità assolutamente diversificate, da regione a regione, da località a località.

In conclusione, 1'aspetto forse più interessante della vicenda della Rsi a Lucca è riscontrabile proprio in relazione ai suoi limiti e all'apparente scarsa importanza della sua parabola amministrativa. Infatti, anche nel caso di un'esperienza pur breve e sfilacciata quale è stata quella del fascismo repubblicano e della Rsi lucchese, si possono comunque rintracciare, almeno nella gestione del primo capo della provincia Piazzesi (settembre 1943- maggio 1944) tensioni e aspirazioni ad un governo effettivo del territorio. Anche nel caso di un'amministrazione Rsi radicalmente e strutturalmente fragile come quella di Lucca, le autorità fasciste e italiane tentarono ripetutamente di rivendicare il loro ruolo, rendendo anche esplicito, in diverse occasioni, il loro disappunto per il comportamento dell'alleato germanico. Infine, come accennato prima, pure nella tensione finale dell'arretramento e della fuga al nord, caratterizzata essenzialmente dalla razzia e della violenza, queste autorità non rinunciarono al tentativo di legittimare e legalizzare la loro azione istituendo commissioni e producendo documentazione amministrativa.

Il fascismo repubblicano, anche se schiacciato dagli eventi e da un alleato ingombrante, non sempre, non nella totalità dei suoi protagonisti percepì se stesso e il suo agire come una semplice marionetta in mano ai comandi tedeschi; questo non avvenne, non completamente almeno, neanche nel caso di una cornice amministrativa fragile e sfilacciata come quella di Lucca. Il fatto che le aspirazioni ad un governo autonomo siano state soprattutto delle "illusioni", dovrebbe forse portare l'attenzione delle future ricerche anche su questo punto: dopo il 25 luglio 1943, dopo 1'arresto di Mussolini, dopo l'evolversi delle vicende belliche (nell'autunno 1943, una vittoria dell'Asse sembrava sempre meno raggiungibile), dopo l'8 settembre, dopo e in considerazione di tutti questi elementi si trovarono e si insediarono ancora uomini, ancora fascisti, disposti a governare e ad amministrare i territori di un nuovo Stato, la cui sopravvivenza e il cui futuro erano assolutamente incerti.

Il presente contributo è una rielaborazione rivista ed ampliata della relazione presentata al convegno "I molti territori della Repubblica fascista. Amministrazione e società nella RSI", Ferrara 27/28 settembre 2017. A fronte dell'impossibilità di sottoporre il testo ad un processo di double blind peer-review, $i$ curatori hanno optato per una revisione preliminare da parte del relativo discussant di sessione. 


\section{Bibliografia}

Adduci N. 2014, Gli altri. Fascismo repubblicano e comunità nel Torinese, 1943-1945, Milano: Franco Angeli.

Baldissara L. 1998, Tecnica e politica dell'amministrazione. Saggio sulle culture amministrative e di governo municipale fra anni Trenta e Cinquanta, Bologna: il Mulino.

Bennati M. 2016a, «I colpevoli di ogni sorta e d'ogni ceto saranno puniti inesorabilmente». Epurazione e punizione dei delitti fascisti in provincia di Lucca (1944-1948), Tesi di laurea magistrale in Storia e civiltà, relatore Luca Baldissara- correlatore Gianluca Fulvetti, Università di Pisa

Bennati M. 2016b, Dalla guerra alla Repubblica: l'epurazione mancata (1944-1947), in Finelli P. e Manfredi M. (eds.) 2016, Lucca 1946. Ricostruzione e formazione di un 'identità politica, Lucca: Pacini Fazzi

Bonomini L. (ed.) 1974, Riservato a Mussolini. Notiziari giornalieri della Guardia Nazionale Repubblicana, novembre 1943-giugno 1944, Milano: Feltrinelli.

Fulvetti G. 2006, Una comunità in guerra. La certosa di Farneta tra Resistenza civile e violenza nazista, Napoli: l'Ancora del Mediterraneo

Fulvetti G. e Pelini F. (eds.) 2006, La politica del massacro. Per un atlante delle stragi naziste in Toscana, Napoli: l'Ancora del Mediterraneo

Fulvetti G. (ed.) 2014, Di fronte all'estremo. Don Aldo Mei, cattolici, chiese, resistenze, Lucca: Pacini Fazzi

Lenzi L. 1995, Primi appunti per una storia della Chiesa di Lucca nel passaggio dal regime fascista alla vita democratica (1943-1946), in Poli F. e Villani G. (eds.) 1995, Chiese Toscane. Cronache di guerra 1940- 1945, Firenze: Libreria Editrice Fiorentina

Mantelli B. 2006, Gli italiani in Germania 1938-1945: un universo ricco di sfumature, "Quaderni Istrevi", 1

Mazzoni M. 2006, La repubblica sociale italiana in Toscana, in Palla M. (ed.) 2006, Storia della Resistenza in Toscana, Roma: Carocci

Osti Guerrazzi A. 2012, Storia della Repubblica sociale italiana, Roma: Carocci

Pardini G. 2001, La Repubblica Sociale Italiana e la guerra in provincia di Lucca (19401945), Lucca: Edizioni San Marco

Pavone C. 1985, Tre governi e due occupazioni, in Ferratini Tosi F., Grassi G. e Legnani M. (eds.) 1988, L'Italia nella seconda guerra mondiale e nella Resistenza, Milano: Franco Angeli

Pavone C. 2006, Una guerra civile. Saggio storico sulla moralità nella Resistenza, Torino: Bollati Boringhieri (ed. or. 1991)

Pesi E. 2013, Dalla guerra alla democrazia. La ricostruzione in provincia di Lucca 19441948, Lucca: Pacini Fazzi

Pesi E. 2010, Resistenze civili. Clero e popolazione lucchese nella seconda guerra mondiale, Lucca: Pacini Fazzi 
Piazzesi M. 1980, Diario di uno squadrista toscano (1919-1922), Roma: Bonacci

Rossi A. 2006, Fascisti toscani nella Repubblica di Salò, Pisa: BFS Edizioni.

Rovatti T. 2011, Leoni vegetariani. La violenza fascista durante la RSI, Bologna: Clueb.

Rovatti T. 2014, Linee di ricerca sulla Repubblica sociale italiana, "Studi storici”, 1. 